\title{
A novel few-shot learning based multi-modality fusion model for COVID-19 rumor detection from online social media
}

\author{
Heng-yang Lu ${ }^{\text {Corresp., } 1,2}$, Chenyou Fan ${ }^{3}$, Xiaoning Song ${ }^{1}$, Wei Fang ${ }^{1}$ \\ 1 Jiangsu Provincial Engineering Laboratory of Pattern Recognition and Computational Intelligence, School of Artificial Intelligence and Computer Science, \\ Jiangnan University, Wuxi, China \\ 2 National Key Laboratory for Novel Software Technology, Nanjing University, Nanjing, China \\ 3 Shenzhen Institute of Artificial Intelligence and Robotics for Society, Shenzhen, China \\ Corresponding Author: Heng-yang Lu \\ Email address: luhengyang@jiangnan.edu.cn
}

Background. Rumor detection is a popular research topic in natural language processing and data mining. Since the outbreak of COVID-19, related rumors have been widely posted and spread on online social media, which have seriously affected people's daily lives, national economy, social stability, etc. It is both theoretically and practically essential to detect and refute COVID-19 rumors fast and effectively. As COVID-19 was an emergent event that was outbreaking drastically, the related rumor instances were so scarce and distinct at its early stage. This makes the detection task a typical few-shot learning problem. However, traditional rumor detection techniques focused on detecting existed events with enough training instances, so that they fail to detect emergent events such as COVID-19. Therefore, developing a new few-shot rumor detection framework has become critical and emergent to prevent outbreaking rumors at early stages.

Methods. This article focuses on few-shot rumor detection, especially for detecting COVID-19 rumors from Sina Weibo with only a minimal number of labeled instances. We contribute a Sina Weibo COVID-19 rumor dataset for few-shot rumor detection and propose a few-shot learning-based multi-modality fusion model for few-shot rumor detection. A full microblog consists of the source post and corresponding comments, which are considered as two modalities and fused with the meta-learning methods.

Results. Experiments of few-shot rumor detection on the collected Weibo dataset and the PHEME public dataset have shown significant improvement and generality of the proposed model. 
1 A novel few-shot learning based multi-modality fusion 2 model for COVID-19 rumor detection from online 3 social media

$7 \quad{ }^{1}$ Jiangsu Provincial Engineering Laboratory of Pattern Recognition and Computational

8 Intelligence, School of Artificial Intelligence and Computer Science, Jiangnan University, Wuxi, 9 China

$10{ }^{2}$ National Key Laboratory for Novel Software Technology, Nanjing University, Nanjing, China

Corresponding Author:

Heng-yang $\mathrm{Lu}^{*}$

No. 1800, Lihu Avenue, Jiangnan University, Wuxi, Jiangsu, 214122, China

Email address: luhengyang@jiangnan.edu.cn 


\section{Abstract}

43 Background. Rumor detection is a popular research topic in natural language processing and 44 data mining. Since the outbreak of COVID-19, related rumors have been widely posted and 45 spread on online social media, which have seriously affected people's daily lives, national 46 economy, social stability, etc. It is both theoretically and practically essential to detect and refute 47 COVID-19 rumors fast and effectively. As COVID-19 was an emergent event that was

48 outbreaking drastically, the related rumor instances were so scarce and distinct at its early stage. 49 This makes the detection task a typical few-shot learning problem. However, traditional rumor 50 detection techniques focused on detecting existed events with enough training instances, so that 51 they fail to detect emergent events such as COVID-19. Therefore, developing a new few-shot rumor detection framework has become critical and emergent to prevent outbreaking rumors at early stages.

Methods. This article focuses on few-shot rumor detection, especially for detecting COVID-19

55 rumors from Sina Weibo with only a minimal number of labeled instances. We contribute a Sina Weibo COVID-19 rumor dataset for few-shot rumor detection and propose a few-shot learningbased multi-modality fusion model for few-shot rumor detection. A full microblog consists of the source post and corresponding comments, which are considered as two modalities and fused with the meta-learning methods.

Results. Experiments of few-shot rumor detection on the collected Weibo dataset and the PHEME public dataset have shown significant improvement and generality of the proposed model.

\section{Introduction}

From the early social psychology literature, a rumor refers to a story or a statement whose truth value is unverified or deliberately false (Allport et al., 1947). More recently, DiFonzo and associates defined rumor as unverified and instrumentally relevant information statements in circulation that arise in contexts of ambiguity and that function primarily to help people make sense and manage threat (DiFonzo et al., 2011). With the fast development of the Internet, the widespread of rumors online has become a major social problem nowadays. Especially on popular online social media such as Sina Weibo and Twitter, users or machines post millions of unverified messages every day. Since the breakout of COVID-19, rumors about COVID-19 have been continuously posted and spread, causing the panic of the public and placing considerable losses on the economy and other aspects of society. Thus, the study of discovering and dispelling rumors fast and accurately has become both theoretically and practically valuable. So, rumor detection on social media has become one of the recently popular research areas. Online social media are naturally suitable for stimulating mass discussions and spreading information. Users usually initialize conversations over spotlighted events/topics and thus generate a series of related posts over the same events/topics. Each conversation/discussion consists of a source post, corresponding replies and reposts. Therefore, most existing works detect rumors on social media at a macro level. They aim to determine whether the public 
82 discussions relating to a certain event/topic belongs to rumor or not (Wu et al., 2015). Existing

83 works under this setting contain both traditional machine learning models with hand-crafted 84 features (Castillo et al., 2011; Yang et al., 2012; Kwon et al. 2013; Jin et al., 2014; Wu et al., 85 2015), and deep learning-based models (Ma et al., 2016; Yu et al., 2017; Chen et al., 2018; Ma et 86 al., 2019; Bian et al., 2020). One of the other research lines aims to detect rumors at a micro 87 level, which means to detect whether a single post belongs to a rumor. It has practical value for 88 those who care more about the credibility of single posts. Pioneer works have been conducted on 89 the Twitter rumor detection task (Sicilia et al., 2017; Sicilia et al., 2018 (a)). Most existing rumor 90 detection models assume that each event has plenty of training instances and regard the task of 91 rumor detection as the classification problem based on supervised learning. Therefore a coherent 92 challenge of existing rumor detection methods is identifying rumors relating to some suddenly happened events such that very few instances were available at the early stages of the events. For the macro-level models, it is a possible solution to set time windows for learning good features (Kwon et al., 2017) at the early stage. It is still based on supervised learning and the discussed events appear in both the training set and test set.

However, the COVID-19 is an emergent event, which has never occurred in the past. This means there is only rarely labeled data for this kind of emergent event at the early stage. In this scenario, all the previous supervised learning-based methods are not applicable, because the training data and the test data belong to distinct events. Previous works on cross-topic rumor detection have discussed this problem, which added knowledge of the test topic in the training set (Sicilia et al., 2018 (b)). According to the conclusion in (Sicilia et al., 2018 (b)), to obtain good results in cross-topic detection, at least $80 \%$ of the test topic knowledge should be included in the training set. Therefore, existing works have huge difficulty in rumor detection for emergent events like COVID-9 with very little labeled data, the main challenges include: (1) The rumors about the target emergent event to be detected has never occurred before, so that the history data of other events could hardly contribute to building prediction models. (2) The number of labeled instances for the target emergent event is extremely scarce, e.g. only 1 or 3 or 5, which makes the popular "pretraining and finetuning" paradigm fail under this situation. Motivated by the necessity of COVID-19 rumor detection under these real challenges, we formulate it as a few-shot learning task. Few-shot learning is able to learn an adaptable model with only a few labeled data. It can predict rumors about emergent events, which have never occurred in the training set. Considering collecting information like the user profile is both timeconsuming and privacy-sensitive, we aim to detect rumors only based on the text contents from online social media. We regard a full microblog consists of two modalities, the source post and the limited number of corresponding comments, and aim to detect whether a full microblog belongs to a rumor. Both modalities are used for building fusion models. To the best of our knowledge, this is the first work tackling the challenge of detecting rumors with very few instances over emergent events and considering the rumor detection task as a few-shot learning task. The main contributions are as follows: 
121

122

123

124

125

126

127

128

129

130

131

132

133

134

135

136

137

138

139

140

141

142

143

144

145

146

147

148

149

150

151

152

153

154

155

156

157

158

159

160

- We collect and contribute a publicly available rumor dataset that is suitable for few-shot learning from Sina Weibo, the largest and most popular online social media in China. This dataset contains 11 COVID-19 irrelevant events and 3 COVID-19 relevant events, which sums to a total of 3,840 instances, of which 1,975 are rumors and 1,865 are non-rumors.

- We propose the novel problem of few-shot rumor detection on online social media. It aims to detect rumors of emergent events, which have never happened, with only a very small number of labeled instances. The definition of instances considers the characteristics of online social media by containing both source posts and corresponding comments.

- We introduce a few-shot learning-based multi-modality fusion model named COMFUSE for COVID-19 rumor detection, including text embeddings modules with pre-trained BERT model, feature extraction module with multilayer Bi-GRUs, multi-modality feature fusion module with a fusion layer, and meta-learning based few-shot learning paradigm for rumor detection. We perform extensive evaluations on benchmark datasets to show that our model is superior to the state-of-the-art baselines in the few-shot situation, which can detect rumors of emergent events with only a small number of labeled instances.

\section{literature review}

This paper focuses on the few-shot rumor detection task on social media for the emergent event like COVID-19, related literature reviews include rumor detection, rumor detection at an early stage, and few-shot learning.

\section{Rumor detection}

Most early works on rumor detect extracted hand-crafted features and built classifiers under supervised learning. For example, Castillo and associates constructed features from the message, user profiles and topics to study the credibility of tweets by applying SVM and Naive Bayes (Castillo et al., 2011). Kwon and associates comprehensively explored the user, structural, linguistic and temporal features in rumor detection tasks (Kwon et al., 2017). Sicilia and associates applied new features such as the likelihood a tweet is retweeted, and the fraction of tweets with URLs to detect health-related rumors (Sicilia et al., 2018 (a)). Besides, hand-crafted features such as location-based features (Yang et al., 2012), temporal features (Kwon et al., 2013), topical space features (Jin et al., 2016) and sentimental features (Liu et al., 2015; Mohammad et al., 2017) are also applied. In this stage, traditional machine learning algorithms such as support vector machines (Yang et al., 2012) and decision trees (Castillo et al., 2011; Zhao et al., 2015) were the common choices. However, hand-crafted feature engineering is timeconsuming and with high labor costs. Benefit from the development of deep learning, deeplearning based features have been widely applied to rumor detection recently. These features are extracted automatically in the form of embeddings by training deep neural networks.

Representative models such as recurrent neural networks (RNNs) and convolutional neural networks (CNNs) are widely used to extract essential features of given texts for rumor detection (Yu et al., 2017; Chen et al., 2018; Ma et al., 2019; Bian et al., 2020). There are also some works considering the characteristics of social media. For the propagation structure in social media, Liu

Peer] Comput. Sci. reviewing PDF | (CS-2021:03:59459:1:1:NEW 13 Jul 2021) 
161 proposed to use the propagation information to help detect rumors (Liu et al., 2016). For the 162 response and reply operations in Twitter, retweets (Yuan et al., 2019) and replies (Ma et al., 163 2019) along with source tweets were utilized.

\section{Early-stage Rumor Detection}

165 Detect rumors at the early stage is both necessary and challenging. A comprehensive study was

166

167

168

169

170

171

172

173

174

175

176

177

178

179

180

181

182

183

184

185

186

187

188

189

190

191

192

193

194

195

196

197

198

199

200

conducted to explore rumor detection performance over varying time windows with four kinds of hand-crafted features include user, structural, linguistic and temporal-based features (Kwon et al., 2017). It reveals that user and linguistic features are suitable for building early detection models and proposed a practical algorithm that does not require full snapshots nor complete historical records. Similar strategies were applied to deep learning-based models such as GANGRU (Ma et al., 2019) and Bi-GCN (Bian et al., 2020), which set a detection delay time and evaluated with tweets posted no later than the delay. These introduced works detect early rumors at the macro level and the detected events of the discussions online have appeared in both the training set and test set. Another pioneer work of early rumor detection focused on cross-topic rumor detection (Sicilia et al., 2018 (b)), which aims to detect rumors about an unseen topic that has never used and existed in the training set. This paper detected rumors at the micro-level and implies that under this practical setting, it requires at least $80 \%$ of the test topic samples to be included in the training set, in order to achieve good results. The cross-topic task was also discussed in a recent proposed work about rumor detection with imbalanced learning (Fard et al., 2020).

\section{Few-shot learning}

Few-shot learning assumes that very few labeled instances are available, which is a challenging task in machine learning (Vinyals et al., 2016; Finn et al., 2017; Snell et al., 2017; Sung et al., 2018). Meta-learning is one of the popular strategies in few-shot learning, developing machine learning models to predict unseen categories with few labeled data. The core idea of metalearning is to learn transferable knowledge on training data that can adapt to new tasks efficiently with just a few examples of the new tasks. Optimization-based meta-learning approaches such as MAML (Finn et al., 2017) aim to search for optimal initial parameters of models which can quickly adapt to new tasks with just a few gradient steps. Meta-transfer learning (MTL) (Sun et al., 2019) proposed to avoid the overfitting problem during training a small amount of data from the unseen category. Metric-based meta-learning approaches such as MatchingNet (Vinyals et al., 2016) and PrototypicalNet (Snell et al., 2017) aim to learn a better feature space to reflect the distance between instances. Although few-shot learning has achieved success in image classification tasks, very few research attempts have been made to study how to detect rumors with few instances.

\section{Materials \& Methods} Problem setting

This paper models the COVID-19 rumor detection problem as a few-shot binary classification task, denoted as $N$-way $M$-event $K$-shot $Q$-query. $N$ refers to the distinct number of few-shot

Peer] Comput. Sci. reviewing PDF | (CS-2021:03:59459:1:1:NEW 13 Jul 2021) 
201 learning labels, which we have $N=2$ in this paper as we consider an instance as rumor or non-

202

203

204

205

206

207

208

209

210

211

212

213

214

215

216

217

218

219

220

221

222

223

224

225

226

227

228

229

230

231

232

233

234

235

236

237

rumor. $M$ represents the number of sampled events among $E$. Let $E=E_{p} \cup E_{s}$ denote a set of given events, where $E_{p}$ refers to those events that happened in the past and have enough labeled instances for training, $E_{S}$ refers to those events that happened suddenly and should be predicted with only a small number of labeled instances. $K$ represents the number of sampled instances in the support set (training set) for each label, and $Q$ represents the number of sampled instances in the query set (test set) for each label.

Each event is composed of a set of related instances. Given an instance $\left(x_{i}, y_{i}\right), x_{i}=\left[m_{i}, c_{i}\right]$, where $x_{i}$ is a full microblog, $m_{i}$ refers to the text content (post) of the $i$-th microblog, and $c_{i}=\left[c_{i 1}, c_{i 2}, \ldots, c_{i l}\right.$ ] consists of the $l$ comments of the $i$-th microblog. We regard $m_{i}$ and $c_{i}$ as two modalities. $y_{i}$ is the label of the $i$-th instance, which indicates whether the $i$-th instance belongs to rumor or not. The few-shot learning target is to train a classifier $\mathbb{C}$ to predict whether an instance in $E_{S}$ belongs to a rumor with only a few numbers of labeled data. Models trained on instances of $E_{p}$ are used for task adaptation.

Data

Sina Weibo is a popular Chinese online social media platform, where users can post or repost, and leave comments with each other. Fig. 1 is a rumor example from Sina Weibo. It mainly contains the post (similar to the source post on Twitter) and corresponding comments (similar to the replies on Twitter). If it is judged as a rumor by the official platform, there is a reminder display on the top of the page.

We construct and share a novel dataset based on Weibo for the research of few-shot rumor detection ${ }^{1}$. The publicly available dataset is written in Chinese and each instance contains a source post along with corresponding comments, the posted date and its label are also included. Our collected dataset contains 11 independent and distinct COVID-19 irrelevant events that happened in the past and 3 COVID-19 relevant events that happened since the breakout of COVID-19. For each event, we crawl related microblogs consisting of source posts (modality 1) and corresponding comments (modality 2) from Sina Weibo, in which both rumors and nonrumors are covered. The event names are used as searching keywords. We provide the corresponding descriptions of all events are as follows (the original names are in Chinese, here we have translated them to English).

- MH370: This event is about the crash of Malaysia Airlines MH370 discussed online.

- College entrance exams: This event is about the annual Chinese college entrance exams.

- Olympics: This event is the discussion about the news of Olympics games on Sina Weibo.

- Urban managers: Urban manager is an occupation in China, who helps keep the city clean and safe. This event is the discussion about how urban managements perform their official duties.

- Cola: This event is about Coke Cola from the perspectives of food additives.

${ }^{1}$ https://github.com/jncsnlp/Sina-Weibo-Rumors-for-few-shot-learning-research 
238

239

240

241

242

243

244

245

246

247

248

249

250

251

252

253

254

255

256

257

258

259

260

261

262

263

264

265

266

267

268

269

270

271

272

273

274

275

- Child trafficking: This event is about child trafficking and asking for help reported on Sina Weibo.

- Waste oil: This event is about the news of waste oil used for cooking from the perspectives of food safety.

- Accident: This event is about accidents that happened and reported on Sina Weibo, such as traffic accidents.

- Earthquake: This event is about the earthquake discussed and reported on Sina Weibo.

- Typhoon: This event is about the typhoon discussed and reported on Sina Weibo.

- Rabies: This event is the discussion about serious death caused by rabies on Sina Weibo.

- Lockdown the city: This event is the discussions about the lock-down-city policy online.

- Zhong Nanshan: This event is about the Chinese anti-epidemic expert Dr. Zhong Nanshan.

- Wuhan: This event is about discussions on the COVID-19 in Wuhan.

The official Sina Weibo community management center ${ }^{2}$ displays all the fake posts judged and labeled by professional human moderators, which is commonly used as the source of collecting Weibo rumors (Ma et al., 2016; Yuan et al., 2019). Fig. 2 illustrated the workflow of the judgement for the rumor displayed in Fig. 1, similar to the process of the court ruling. The final judgement by the official platform (on the top of Fig. 2) comes from both reported reasons from other users (on the bottom left) and explanations from the posted user (on the bottom right). Once the post is labeled as a rumor, a "Fake post" (the original one is in Chinese) sign would appear on the posted page, as Fig. 1 shows. We implement a web crawler to collect all the reported posts from the official Sina Weibo community management center, posted date starts from May 2012 to December 2020. Keywords of distinct events (original formats are in Chinese, translated to English in Table 1) are then applied to filter event-related instances as rumors. To collect non-rumors, we choose the same keywords used for collecting rumors of selected events. The web crawler is designed to search and crawl the posts with given keywords. For those crawled posts which are not marked as "Fake post" by the official platform, we take them as non-rumors. All the corresponding comments are crawled together.

Due to the repost operation in Sina Weibo, which is similar to the re-tweet feature in Twitter, there exist duplications in the originally collected data. We exploit Hamming distance to filter similar or repetitive texts. Specifically, we treat two source posts with hamming distance less than a threshold (e.g., 6) as duplicates and just retain one of them in the dataset. After this deduplication operation, the statistics of the Weibo dataset are as Table 1 shows.

\section{Few-shot Rumor detection}

The general overflow of COMFUSE is as Fig. 3 shows. The input microblogs consist of source posts along with corresponding comments. Firstly, the pre-trained Bidirectional Encoder Representations from Transformers model (BERT) is applied to achieve the word embeddings of the input microblogs. Then two bidirectional GRUs are used to learn features of source posts and comments separately. A fusion layer is applied to fuse the features of both modalities, which are

2 https://service.account.weibo.com/ 
276 source posts and comments. Finally, meta-learning is applied to detect rumors related to new 277 events with task adaptation.

278 Pretrained word embeddings. Recently, transformer-based NLP models (Vaswani et al., 2017) 279 have shown that attention-based embedding mechanisms have great superiority over simple 280 structured embedding models (Sun et al., 2019), such as word2vec (Mikolov et al., 2013) and 281 GloVe (Pennington et al., 2014). In this paper, we utilize BERT models pre-trained on large282 scale such as Wikipedia with Transformers to embed the inputs. Given an instance $x_{i}=\left[m_{i}, c_{i 1}\right.$ $\left.283, \ldots, c_{i l}\right]$, both the source posts and comments are in the format of sequences. Fig. 4 demonstrates 284 embedding inputs with pre-trained BERT in detail. For an input (a post $m_{i}$ or a comment $c_{i l}$ ), the 285 first step is tokenization based on the predefined vocabulary and achieve $\left[t_{1}, t_{2}, \ldots, t_{n}\right]$, where $n$ 286 is the number of tokens. An embedding layer is then applied to achieve initialized embeddings $e_{k}$ 287 for every token $t_{k}$ and achieve $\left[e_{1}, e_{2}, \ldots, e_{n}\right]$. Then, the embeddings $B=\left[b_{1}, b_{2}, \ldots, b_{n}\right]$ become 288 the output with transformer models. For the given input $x_{i}=\left[m_{i}, c_{i 1}, \ldots, c_{i l}\right]$, the outputs of this procedure are corresponding pre-trained BERT embeddings, denoted as $\left[B^{m_{i}}, B^{c_{i 1}}, \ldots, B^{c_{i l}}\right]$.

290 Bi-GRUs feature extractions. Recently, it is the mainstream to extract features from texts with 291 deep neural networks. Representative RNNs-based models such as LSTMs and GRUs have 292 shown effectiveness in the rumor detection task (Liu et al., 2019; Wang et al., 2020). In this 293 paper, we apply bidirectional GRUs (Bi-GRUs) to extract features of source posts and 294 corresponding comments. We take the post $m$ as an example. After applying pretrained BERT 295 embeddings, the input post $m$ turns to the embeddings matrix $B^{m}=\left[b_{1}^{m}, b_{2}^{m}, \ldots, b_{n}^{m}\right]$, where $n$ is 296 the same definition of token numbers. The BiGRUs are applied upon the embedding matrix to 297 further decode post $m$ to textual hidden features denoted as $H^{m}=\left[h_{1}^{m}, h_{2}^{m}, \ldots, h_{n}^{m}\right]$. The general 298 structure of Bi-GRUs is as Fig. 5 shows.

299 For the $j$-th input embeddings $b_{j}^{m}$, the decoded features $h_{j}^{m}$ of Bi-GRUs' outcome in one 300 direction can be denoted as $h_{j}^{m}=G R U s\left(b_{j}^{m}, h_{j-1}^{m}\right)$. In Equations (1) - (4) we show its complete 301 form in detail:

$$
\begin{gathered}
r_{j}^{m}=\sigma\left(W_{r} b_{j}^{m}+\beta_{r}+W_{h r} h_{j-1}^{m}+\beta_{h r}\right) .(1) \\
z_{j}^{m}=\sigma\left(W_{z} b_{j}^{m}+\beta_{z}+W_{h z} h_{j-1}^{m}+\beta_{h z}\right) .(2) \\
h_{j}^{m^{\prime}}=\tanh \left(W_{h} b_{j}^{m}+\beta_{h}+r_{j}^{m} *\left(W_{h h_{j-1}}^{m}+\beta_{h h}\right)\right) . \\
h_{j}^{m}=\left(1-z_{j}^{m}\right) * h_{j}^{m^{\prime}}+z_{j}^{m} * h_{j-1}^{m} \cdot \text { (4) }
\end{gathered}
$$

306

307

The hidden states of the forward input sequence with $n$ tokens can be represented as $\overrightarrow{H^{m}}=G R U S$ $\left(\overrightarrow{B^{m}}, h_{0}\right)$, where $h_{0}$ is the initial hidden state. Similarly, the hidden states of the backward input 308 sequence are represented as $\overleftarrow{H^{m}}=G R U S\left(\overleftarrow{B^{m}}, h_{0}\right)$. The final hidden states of both directions are 309 310 calculated as Equation (5) shows, which are also regarded as the features for further rumor detection. 


$$
H^{m}=\frac{\left(\overrightarrow{H^{m}}+\overleftrightarrow{H^{m}}\right)}{2}
$$

312 For the $i$-th microblog, the extracted feature of the post $m_{i}$ is denoted as $H^{m_{i}}$, similarly, the

313 extracted features of comments $\left[c_{i 1}, c_{i 2}, \ldots, c_{i l}\right]$ are denoted as $H^{c^{i}}=\left[H_{1}^{c_{i}, \ldots, H_{l}{ }_{l}}\right]$.

314 Feature fusion layer. Take the $i$-th instance from Sina Weibo for example, we consider both

315

316

317

318

319

320

321

322

323

324

325

326

327

328

329

330

331

332

333

334

335

336

337

338

339

340

341

342

343

344

345 source post $m_{i}$ and corresponding comments $\left[c_{i 1}, \ldots, c_{i l}\right]$ as two modalities. Because each instance may have more than one comment, the fusion layer contains two main steps. The first step is to fuse the features $\left[H_{1}^{{ }^{c}}, \ldots, H_{l}{ }^{i}\right]$ of all comments, denoted as $H^{c}{ }^{i}$. The second step is to fuse the features of the source post and comments.

In the first fusion step, the features of all the $l$ comments in the same instance are extracted via the same Bi-GRUs. As the comments of each microblog are embedded into the same feature space, it is natural to fuse them with the weighted sum of their features. We regard the contribution of each comment to be equal for the rumor detection task, which is defined in Equation (6).

$$
H^{c}{ }^{i}=\sum_{j=1}^{l} H_{j}^{c}
$$

In the second fusion step, the features of the source post and comments in the same instance are extracted via two individual Bi-GRUs. Following the common practice, we fuse these multimodal features together with concatenation to build the features of the microblogs. For the $i$-th instance, the fused feature is defined as $H^{i}=\left[H^{{ }^{m}} ; H^{c}{ }^{c}\right]$.

Few-shot learning. Usually, rumors from online social media are usually produced according to certain events. Rumors of emergent events could be very distinct from events collected in the past, so that rumor detection models could barely generalize on new events. However, breaking events like COVID-19 are unprecedented so that very rare instances are available. This may result in the failure or overfitting to directly build a rumor detection model based on supervised learning with the lack of labeled training data for emergent events.

To tackle this challenge, we propose a few-shot learning paradigm by learning a generic model with labeled data from past observed events and adapting to unseen events with only a few labeled instances. We propose a meta-learning based strategy in learning the few-shot rumor detection tasks. The core idea is to sample a large number of task combinations in training instances so that the model can learn the transferable knowledge for unseen categories. State-ofthe-art methods are optimization-based with the idea of training a good initialized model which could adapt to unseen categories with only a few gradient steps (Finn et al., 2017; Sun et al., 2019).

The learning target of few-shot rumor detection with meta-learning methods is to minimize the adaptation loss on unseen tasks during training. Given a batch of few-shot tasks $\mathcal{B}=\left\{T_{1}, \ldots, T_{|\mathcal{B}|}\right\}$, the total loss $\mathcal{L}$ is calculated as Equation (7) shows. 


$$
w^{*}=\min _{w_{m}, w_{c}} \mathcal{L}\left(w_{m}, w_{c}\right), \text { s.t. } \mathcal{L}\left(w_{m}, w_{c}\right)=\frac{1}{|\mathcal{B}|} \sum_{T \in \mathcal{B}} L_{T}\left(w_{m}, w_{c}\right),(7)
$$

347

348

349

350

351

352

353

354

355

356

357

358

359

360

361

362

363

364

365

366

367

368

369

370

371

372

373

374

375

376

377

378

379

380

\section{8}

79

where $w_{m}$ refers to the parameters of the defined Bi-GRUs for dealing with the modality of source posts and $w_{c}$ refers to the parameters of the defined Bi-GRUs for the modality of comments. $L_{T}$ is the loss of task $T$ and $w^{*}$ is the optimized model which can fast adapt to unseen events. This optimization problem can be solved iteratively with the steps as shown in Fig.6, in order to train the models to adapt to sampled new tasks well. We will demonstrate each of the meta-learning steps in detail.

The COVID-19 rumor detection is defined as the $\boldsymbol{N}$-way $\boldsymbol{M}$-event $\boldsymbol{K}$-shot $\boldsymbol{Q}$-query few-shot learning task.

Step 1. Sampling: This step aims to sample a few-shot task $T$ from $E_{p}$ (events happened in the past). Each event has both rumor and non-rumor instances, which means the sampling times from $E_{p}$ equal to $N \times M$. For an $N$-way $M$-event $K$-shot $Q$-query few-shot learning task $T, K$ rumor instances and $K$ non-rumor instances are sampled from $M$ events respectively to compose of a support set, denoted as $T^{(s)}$. $Q$ rumor and non-rumor instances are also sampled from the same $M$ events respectively to compose of a query set, denoted as $T^{(q)}$. This step would sample $N \times M \times(K+Q)$ instances for task $T$.

Step 2. Adaptation: This step aims to learn latent semantics in unseen categories by adapting the current model to the sampled task $T$ in step 1. This step updates the model parameters $w_{m}$ and $w_{c}$ with the few-shot labeled data in $T^{(s)}$ by performing Stochastic Gradient Descent (SGD), as Equations (8) and (9) shows.

$$
\begin{array}{r}
w_{m}^{\prime}=w_{m}-\alpha \nabla_{w_{m} L_{T}^{(s)}}\left(w_{m}\right), \\
w_{c}^{\prime}=w_{c}-\alpha \nabla_{w_{c} L_{T}(s)}\left(w_{c}\right),(9)
\end{array}
$$

where $\alpha$ is the step size of adaption, $w_{m}^{\prime}$ and $w_{c}^{\prime}$ are parameters of the adapted models, which can extract features of source posts and comments in the query set $T^{(q)}$ for further rumor detection. Step 3. Optimization: This step aims to evaluate $w_{m}^{\prime}$ and $w_{c}^{\prime}$ with more samples in the query set $T^{(q)}$. The empirical loss functions are as Equations (10) and (11) show.

$$
\begin{gathered}
L_{T}\left(w_{m}\right)=L_{T}^{(q)}\left(w_{m}^{\prime}\right)=L_{T}(q) \\
L_{T}\left(w_{c}\right)=L_{T^{(q)}}\left(w_{c}^{\prime}\right)=L_{T^{(q)}}\left(w_{c}-\alpha \nabla_{w_{m} L_{w_{c}} L_{T}(s)}\left(w_{m}\right)\right),
\end{gathered}
$$

To search for the optimal $w_{m}$ and $w_{c}$ defined in Equation (7), we need to compute the Hessian. However, considering the tradeoff between the computational costs and performance, we solve this problem with just one gradient descent to approximate the parameter updates (Finn et al., 2017; Sung et al., 2018).

$$
\begin{gathered}
w_{m} \leftarrow w_{m}-\gamma \nabla_{w_{m}} L_{T}\left(w_{m}\right), \\
w_{c} \leftarrow w_{c}-\gamma \nabla_{w_{c}} L_{T}\left(w_{c}\right),
\end{gathered}
$$

where $\gamma$ is the learning rate. 
381 To detect rumors about suddenly happened events $E_{s}$, we can apply the parameters of the 382 adapted models $w_{m}^{\prime}$ and $w_{c}^{\prime}$ to calculate the probability of the instances with the Sigmoid 383 function.

384

\section{Experiments and Results}

\section{Datasets for experiments}

We carry on extensive empirical studies on two real-world datasets with user comments that have been classified as rumors or non-rumors. The first dataset is collected from Sina Weibo, which is used for detecting rumors about COVID-19. The other dataset we use is PHEME (Zubiaga et al., 2016), which is publicly available and widely used in most rumor detection researches. Details of both datasets are as follows.

Weibo Dataset: We collect microblogs that are written in Chinese from Sina Weibo - the largest online social media in China. In this dataset, there are 14 events with 3,840 instances in total. For each event, both rumors and non-rumors are included. Each event is a hot topic such as MH370, COVID-19 expert Zhong Nanshan, etc., which are widely discussed online. Each instance is recorded with a source post along with its comments. To evaluate the performance of few-shot learning on COVID-19 rumor detection, 11 COVID-19 irrelevant events are selected as the training and validation set, and 3 COVID-19 relevant events are used for testing (listed in Table 1).

PHEME Dataset: This is a publicly available dataset ${ }^{3}$ with tweets from Twitter in English, which is widely used for the evaluation of rumor detection tasks (Zubiaga et al., 2017; Ma et al. 2019). It is collected according to 5 breaking events discussed on Twitter (Zubiaga et al., 2016). Each instance is recorded with a source tweet along with its reply reactions. To evaluate the performance under the settings of few-shot learning, 3 breaking events that happened earlier are selected as the training and validation set (\#Ferguson unrest, \#Ottawa shooting, \#Sydney siege), and the rest 2 events that happened most recently are used for testing (\#Charlie Hebdo shooting, \#Germanwings plane crash). The pre-processing of the PHEME dataset follows the practice in previous work (Ma et al., 2019).

For the Weibo dataset, we crawl the comments of microblogs directly as they are readily available on the same webpage with the source posts. For the PHEME dataset, we regard the replies in the given dataset as comments. For the sake of generality, we randomly divide the dataset into training and validation sets according to distinct events, and repeat three times to form three different splits for robust cross-validation. We choose the number of splits as three for the following reasons. In few-shot learning, the number of splits depends on the number of new events in the test set. We take the 2-way 3-event 5-shot 9-query Weibo dataset for example. It has 3 COVID-19 relevant events to be detected with only a few labeled data. The number of the event in the definition is determined by the number of new events in the test dataset, so it is 3-event. The number of ways indicates the number of labels, which are rumor and non-rumor. With this definition, during the

${ }^{3}$ https://figshare.com/articles/PHEME_dataset_of_rumours_and_non-rumours/4010619 
419 few-shot learning training process, every training epoch will sample 3 different events in the training set, 420 for each event, 5 rumor instances and 5 non-rumor instances will be sampled for training. According to 421 the few-shot learning setting, we guarantee that all events in the training sets should NOT appeared in the 422 testing sets, and vice versa, to avoid the leakage of event information and guarantee that we are testing on complete novel events. We also assume that the number of events in the training set should be no less than the number of events in the test set to ensure the model capacity for adapting to new events. According to our assumption and task settings, we split our Weibo dataset to 3 events (COVID-19 relevant) for testing, and 11 events (COVID-19 irrelevant) for training. We fix the 3 events (COVID-19 relevant) for testing, and construct 3 folds for "cross-validation" over 11 training events (COVID-19 irrelevant) to guarantee that each fold has more than 3 events in the Weibo dataset. Table 2 displays the statistics of the data for experiments.

\section{Baselines for comparisons}

431 Five baselines are selected to compare the performance of few-shot rumor detection, including

432

433

434

435

436

437

438

439

440

441

442

443

444

445

446

447

448

449

450

451

452

453

454

455

456

457

458

459 traditional methods, deep learning methods, and few-shot learning methods.

1. DT-EMB: This baseline model uses the decision tree as the basic classifier, which was applied in traditional rumor detection tasks (Zhao et al., 2015). The feature of each instance is represented by the embeddings encoded by the same pre-trained BERT model.

2. SEQ-CNNs: This deep learning-based baseline trains classification model with features extracted by CNNs, which is a common choice for rumor detection in recent researches $(\mathrm{Yu}$ et al., 2017), the input sequence is encoded by the same BERT pretrained model for fair comparisons.

3. SEQ-Bi-GRUs: This is also a deep learning-based baseline for rumor detection. Bi-GRUs are applied to extract features for training and prediction (Ma et al., 2016; Chen et al., 2018), the input sequence is encoded by the same BERT pretrained model for fair comparisons.

4. GAN-GRU-early: The basic model of this baseline is a popular model named GAN-GRU (Ma et al. 2019). According to the early detection setting in this paper, for each source post, only the latest 3 comments are used for evaluation, which is as same as modality 2 in COMFUSE.

5. BiGCN-early: The basic model of this baseline is a state-of-the-art model named BiGCN (Bian et al. 2020). According to the early detection setting in this paper, for each source post, only the latest 3 comments are used for evaluation, which is as same as modality 2 in COMFUSE.

6. COMFUSE-post-only: This is a simplified model of COMFUSE for ablation study. Only the source post of each microblog is used for training and prediction in the few-shot rumor detection task.

7. COMFUSE-com-only: This is another simplified model of COMFUSE for ablation study. Only the comments of each microblog are used for training and prediction in the few-shot rumor detection task.

The problem setting of this paper is few-shot rumor detection, which assumes that the events in the test set have not occurred in the training set and only a small number of labeled instances is available. DT-EMB, SEQ-CNNs, SEQ-Bi-GRUs, GAN-GRU-early and BiGCN-early are five

PeerJ Comput. Sci. reviewing PDF | (CS-2021:03:59459:1:1:NEW 13 Jul 2021) 
460 baselines for common rumor detections, which require the training set and test set to share the 461 same events. To have fair comparisons, new paradigms are designed for training and testing 462 these baselines. For the traditional machine learning-based model DT-EMB, a small number of 463 labeled data sampled from the new events are put into the training set for training. For SEQ464 CNNs and SEQ-Bi-GRUs, we train the rumor detection model with the training data firstly and 465 finetune the model with a small number of labeled data sampled from the new events. Because

466

467

468

469

470

471

472

473

474

475

476

477

478

479

480

481

482

483

484

485

486

487

488

489

490

491

492

493

494

495

496

497 the original GAN-GRU and BiGCN are not designed as the few-shot learning models, we use the same instances of new events, which are also used for task adaption in COMFUSE for training. For all models, the same random seed is set for sampling and these sampled data do not appear in the test set.

\section{Experimental settings}

According to the problem setting and considering the number of events in the Weibo dataset and PHEME dataset, we define the few-shot rumor detection for the Weibo dataset as 2-way 3-event 5-shot 9-query, for PHEME dataset as 2-way 2-event 5-shot 9-query respectively. We implement COMFUSE with Pytorch 1.8.1 and utilize the pre-trained BERT model from HuggingFace 4 to encode the inputs. We use the uncased Chinese model and uncased English model for the Weibo dataset and PHEME dataset respectively. The source code will be publicly available.

To determine the pad size of the input posts and comments, the statistics of the length per text are performed. The histograms of the Weibo and PHEME datasets are as Fig. 7 and Fig. 8 show. Considering the trade-off between performance and speed, we set the pad size of posts/source tweets as 100 (for Weibo) and 48 (for PHEME) respectively. We set the pad size of comments/replies as 32 for both datasets. Further experiments are conducted to show the influence of different pad size choices. The experimental results of the Weibo dataset are as Fig. 9 and Fig. 10 show.

Fig. 9 displays the results of different pad sizes of source posts with a fixed pad size of comments as 32 on the Weibo dataset. Fig. 10 displays the results of different pad sizes of comments with a fixed pad size of source posts as 100 . Both $x$-axis refer to the pad size and $y$ axis refers to the accuracy performance. We can observe that the rumor detection results of COMFUSE with different pad sizes of posts and comments vary slightly. For the Weibo dataset, the experimental results reveal that it is relatively better to set the pad size as 100 for posts and 32 for comments, which is consistent with our decision based on the statistics of the length in Fig. 7 and 8.

In this paper, we define an instance as $x_{i}=\left[m_{i}, c_{i 1}, \ldots, c_{i l}\right]$, which contains $l$ comments. As we consider few-shot learning scenarios, we assume that there are very few useful comments available in the early stage of an event. Thus, in our experiments, we set the number of relevant comments $l$ as 3 for all experiments, in order to simulate the emerging situations and examine whether our approach can successfully detect rumors from very few labeled instances and informative comments.

${ }^{4}$ https://huggingface.co/ 
498 Performance of few-shot rumor detection

499 In this paper, we treat the rumor detection task as a binary classification problem, and we use

500 classification accuracy as the evaluation metric for comparisons. We conduct experiments on all

501 three splits and examine their averaged performance, as shown in Table 3 and Table 4.

502

503

\section{Discussion}

504

505

506

507

508

509

510

511

512

513

514

515

516

517

518

519

520

521

522

523

524

525

526

527

528

529

530

531

532

533

534

535

536

537

Table 3 displays the performance of COVID-19 rumor detection under the few-shot learning setting. A higher classification accuracy indicates a better performance. It can be observed that the traditional machine learning-based method DT-EMB performs poorly in few-shot rumor detection: it achieves only $56.93 \%$ accuracy on average, which is barely better than random guessing in a binary classification task.

Two state-of-the-art deep learning-based methods SEQ-CNNs and SEQ-Bi-GRUs achieve similar performance around $68 \%$. They significantly improve the detection over traditional DTEMB. One of the reasons is the superior ability of deep neural networks to extract important features from contexts, which contribute to the training of models. Furthermore, the paradigm of pretraining first and then finetuning can optimize the model to fit the data of new events to some extent. However, the number of labeled instances of the unseen events for finetuning is quite small in the few-shot rumor detection task, which may result in the underfitting of the fine-tuned model.

GAN-GRU-early and BiGCN-early are another two SOTA baselines and have reported great performance in the traditional rumor detection task, which the events (topics) appear in both the training set and test set. When applying to the emergent rumor detection scenarios, which assumes the events in the test set have never appeared in the training set, the supervised-based GAN-GRU-early and BiGCN-early models show their limitations and are not suitable for the few-shot rumor detection task. One possible reason that GAN-GRU-early underperforms significantly may because there are scarce instances related to the emergent events in the test set being fed to the generators in the training process. This makes the features extracted in the inference pross hardly reflect the instances related to emergent events. COMFUSE is our proposed multi-modality fusion model for few-shot rumor detection based on the meta-learning approach, with COMFUSE-post-only and COMFUSE-com-only as two simplified versions. COMFUSE-post-only only uses the source posts (source tweets in Twitter) as inputs, as same as DT-EMB, SEQ-CNNs, and SEQ-Bi-GRUs, which are commonly used in existing rumor detection models. Compared with SEQ-CNNs and SEQ-Bi-GRUs, COMFUSEpost-only further improves the few-shot COVID-19 rumor detection accuracy by around 6\%. This shows the effectiveness of applying meta-learning methods with only a small number of labeled data to detect rumors of unseen events. COMFUSE takes advantage of both source posts and corresponding comments of the full microblogs to contribute to the detection of rumors from online social media. Intuitively, comments or replies reflect the positive or negative attitudes of the public towards the source posts, so that should provide additional hints towards judging the credibility of the source posts. 
538 COMFUSE-com-only is also an ablation model, which only uses the comments of instances for

539

540

541

542

543

544

545

546

547

548

549

550

551

552

553

554

555

556

557

558

559

560

561

562

563

564

565

566

567

568

569

570

571

572

573

574

575

576

577

rumor detection. It can be observed that the proposed multi-modality fusion model COMFUSE performs much better than two ablation models COMFUSE-post-only and COMFUSE-comonly, with accuracy improvement by $5 \%$. This shows the necessity of fusing both source posts and comments for rumor detection. Comparing the proposed COMFUSE model with traditional machine learning-based and deep learning-based rumor detection models, it achieves the improvements by $21 \%$ and $10 \%$ respectively, which shows the superiority of the meta-learning based fusion model for few-shot COVID-19 rumor detection.

Table 4 is the experimental results on the public and commonly used rumor dataset PHEME, to show the generality of COMFUSE. The proposed COMFUSE model also achieved the best performance among all the baselines. As the Weibo dataset has more events and instances available during meta-training, the model can be trained with more and diverse event combinations and thus be more capable of adapting to novel events by learning to capture distinct hints for rumor detection. In contrast, we have to use only 3 events in PHEME training and thus may fail to understand the most distinguishing hints in rumors of the PHEME dataset. This explains why the improvement on the PHEME dataset is not as significant as that on the Weibo dataset.

\section{Conclusions}

This paper focuses on the few-shot rumor-detection for unexpected and emergent events which have never or rarely happened before, such as COVID-19. Different from rumor detection on daily events in previous work, emergent events outbreak in sudden so that very few labeled instances can be used for training rumor detection models. As existing rumor detection works assume the events to be predicted are as same as those to be trained, they are greatly limited in rumor detection for emergent events. This paper identifies the rumor detection for emergent events as the few-shot learning tasks, and proposes a few-shot learning-based multi-modality fusion model named COMFUSE to detect COVID-19 rumors in Sina Weibo. It exploits the meta-training methodology to empower the model to adapt to new events with few instances, as well as fully utilizing two modalities including source posts and comments from the online social media to support the detection of rumors. Experiments on our self-collected Weibo dataset and the publicly available PHEME dataset have shown significant improvement on the COVID-19 few-shot rumor detection task and the generalization capacity of the proposed model.

\section{Acknowledgements}

We would like to sincerely thank our colleagues, Professor Xiao-jun Wu, and Jun Sun from Jiangnan University, Professor Jun-yuan Xie, and Chong-jun Wang from Nanjing University, for their kind support and guidance.

\section{References}

\footnotetext{
Allport, G. W, and Postman, L. The psychology of rumor. 1947.
} 
578 Bian T, Xiao X, Xu T, Zhao P, Huang W, Rong Y and Huang J. Rumor Detection on Social

579 Media with Bi-Directional Graph Convolutional Networks[C]. Proceedings of the AAAI

580 Conference on Artificial Intelligence. 2020, 34(01): 549-556.

581 Castillo C, Mendoza M, Poblete B. Information credibility on twitter[C]. Proceedings of the

582 20th international conference on World wide web. 2011: 675-684.

583 Chen T, Li X, Yin $\mathbf{H}$ and Zhang $\mathbf{J}$. Call attention to rumors: Deep attention based recurrent

584 neural networks for early rumor detection[C]. Pacific-Asia conference on knowledge discovery

585 and data mining. Springer, Cham, 2018: 40-52.

586 Derczynski L, Bontcheva K, Liakata M, Procter R, Hoi G W S and Zubiaga A. SemEval-

5872017 Task 8: RumourEval: Determining rumour veracity and support for rumours[C].

588 Proceedings of the 11th International Workshop on Semantic Evaluation (SemEval-2017). 2017:

$58969-76$.

590 DiFonzo, N and Bordia, P. Rumors influence: Toward a dynamic social impact theory of

591 rumor. Psychology Press. 2011: 271-295.

592 Fard AE, Mohammadi M and van de Walle B. Detecting Rumours in Disasters: An

593 Imbalanced Learning Approach. In International Conference on Computational Science, 2020 :

594 639-652.

595 Finn C, Abbeel P, Levine S. Model-agnostic meta-learning for fast adaptation of deep

596 networks[C]. Proceedings of the 34th International Conference on Machine Learning-Volume

597 70. 2017: 1126-1135.

598 Jin Z, Cao J, Jiang YG and Zhang Y. News credibility evaluation on microblog with a

599 hierarchical propagation model. In 2014 IEEE International Conference on Data Mining, 2014:

$600 \quad 230-239$.

601 Jin Z, Cao J, Zhang Y, and Luo J. News verification by exploiting conflicting social

602 viewpoints in microblogs[C]. Proceedings of the AAAI Conference on Artificial Intelligence.

$6032016,30(1)$.

604 Kwon S, Cha M, Jung K, Chen W and Wang Y. Prominent features of rumor propagation in

605 online social media. In 2013 IEEE 13th international conference on data mining, 2013: 1103-

6061108.

607 Kwon, S, Cha, M and Jung, K. Rumor detection over varying time windows. PloS one. 2017, 608 12(1), e0168344.

609 Liu X, Nourbakhsh A, Li Q, Fang R and Shah S. Real-time rumor debunking on twitter. In

610 Proceedings of the 24th ACM international on conference on information and knowledge

611 management, 2015: 1867-1870.

612 Liu Y, Jin X, Shen H. Towards early identification of online rumors based on long short-term

613 memory networks[J]. Information Processing \& Management, 2019, 56(4): 1457-1467.

614 Liu Y, Xu S. Detecting rumors through modeling information propagation networks in a social

615 media environment[J]. IEEE Transactions on computational social systems, 2016, 3(2): 46-62. 
616 Ma J, Gao W, Mitra P, Kwon S, Jansen B J, Wong K F and Cha M. Detecting rumors from 617 microblogs with recurrent neural networks[C]. 25th International Joint Conference on Artificial 618 Intelligence, 2016: 3818-3824.

619 Ma J, Gao W, Wong K F. Detect rumors on twitter by promoting information campaigns with 620 generative adversarial learning[C]. The World Wide Web Conference. 2019: 3049-3055.

621 Mikolov T, Sutskever I, Chen K, Corrado G S and Dean J. Distributed Representations of

622 Words and Phrases and their Compositionality[C]. Proceedings of the 27th NeurIPS Conference 623 on Neural Information Processing Systems, 2013: 3111-3119.

624 Mohammad S M, Sobhani P and Kiritchenko S. Stance and sentiment in tweets[J]. ACM 625 Transactions on Internet Technology (TOIT), 2017, 17(3): 1-23.

626 Pennington J, Richard S and Christopher D. M. Glove: Global vectors for word

627 representation[C]. In Proceedings of the 2014 conference on empirical methods in natural 628 language processing, 2014: 1532-1543.

629 Sicilia R, Giudice SL, Pei Y, Pechenizkiy M and Soda P. Health-related rumour detection on 630 Twitter. In 2017 IEEE International Conference on Bioinformatics and Biomedicine (BIBM), 631 2017: 1599-1606.

632 Sicilia R (a), Giudice SL, Pei Y, Pechenizkiy M and Soda P. Twitter rumour detection in the 633 health domain. Expert Systems with Applications, 2018, 110: 33-40.

634 Sicilia R (b), Merone M, Valenti R, Cordelli E, D’Antoni F, De Ruvo V, and Soda P. Cross635 topic rumour detection in the health domain. In 2018 IEEE International Conference on 636 Bioinformatics and Biomedicine (BIBM), 2018: 2056-2063.

637 Sung F, Yang Y, Zhang L, Xiang T, Torr P H and Hospedales T M. Learning to compare: 638 Relation network for few-shot learning[C]. Proceedings of the IEEE Conference on Computer 639 Vision and Pattern Recognition. 2018: 1199-1208.

640 Snell J, Swersky K, Zemel R. Prototypical networks for few-shot learning[C]. Advances in 641 neural information processing systems. 2017: 4077-4087.

642 Sun Q, Liu Y, Chua T S and Schiele B. Meta-transfer learning for few-shot learning[C].

643 Proceedings of the IEEE conference on computer vision and pattern recognition. 2019: 403-412.

644 Sun F, Liu J, Wu J, Pei C, Lin X, Ou W and Jiang P. BERT4Rec: Sequential

645 recommendation with bidirectional encoder representations from transformer[C]. Proceedings of 646 the 28th ACM international conference on information and knowledge management. 2019: 14416471450.

648 Vaswani A, Shazeer N, Parmar N Uszkoreit J, Jones L, Gomez A N and Polosukhin I.

649 Attention is all you need[C]. Proceedings of the 31 st International Conference on Neural 650 Information Processing Systems. 2017: 6000-6010.

651 Vinyals O, Blundell C, Lillicrap T and Wierstra D. Matching networks for one shot 652 learning[J]. Advances in neural information processing systems, 2016, 29: 3630-3638.

653 Wang Z, Guo Y. Empower rumor events detection from Chinese microblogs with multi-type 654 individual information[J]. Knowledge and Information Systems, 2020, 62(9): 3585-3614. 
655 Wu K, Yang S and Zhu KQ. False rumors detection on sina weibo by propagation structures. In 2015 IEEE 31st international conference on data engineering, 2015: 651-662.

657 Yang F, Liu Y, Yu X and Yang M. Automatic detection of rumor on sina weibo. In 658 Proceedings of the ACM SIGKDD workshop on mining data semantics, 2012: 1-7.

659 Yuan C, Ma Q, Zhou W, Han J and Hu S. Jointly embedding the local and global relations of 660 heterogeneous graph for rumor detection[C]. 2019 IEEE International Conference on Data 661 Mining (ICDM). IEEE, 2019: 796-805.

662 Yu F, Liu Q, Wu S, Wang $L$ and Tan T. A convolutional approach for misinformation 663 identification[C]. Proceedings of the 26th International Joint Conference on Artificial 664 Intelligence. 2017: 3901-3907.

665 Zhao Z, Resnick P, Mei Q. Enquiring minds: Early detection of rumors in social media from 666 enquiry posts[C]. Proceedings of the 24th international conference on world wide web. 2015: 667 1395-1405.

668 Zubiaga A, Liakata M, Procter R. Learning reporting dynamics during breaking news for 669 rumour detection in social media[J]. arXiv preprint arXiv:1610.07363, 2016.

670 Zubiaga A, Liakata M, Procter R, Wong Sak Hoi G and Tolmie P. Analysing How People

671 Orient to and Spread Rumours in Social Media by Looking at Conversational Threads[J]. PLoS 672 ONE, 2016, 11(3): 1-29.

673 Zubiaga A, Liakata M, Procter R. Exploiting context for rumour detection in social media[C]. 674 International Conference on Social Informatics. Springer, Cham, 2017: 109-123. 


\section{Table $\mathbf{1}$ (on next page)}

Table 1 Statistics of Events for the COVID-19 Rumor Dataset After Removing Duplicates 
1 Table 1 Statistics of Events for the COVID-19 Rumor Dataset After Removing Duplicates 2

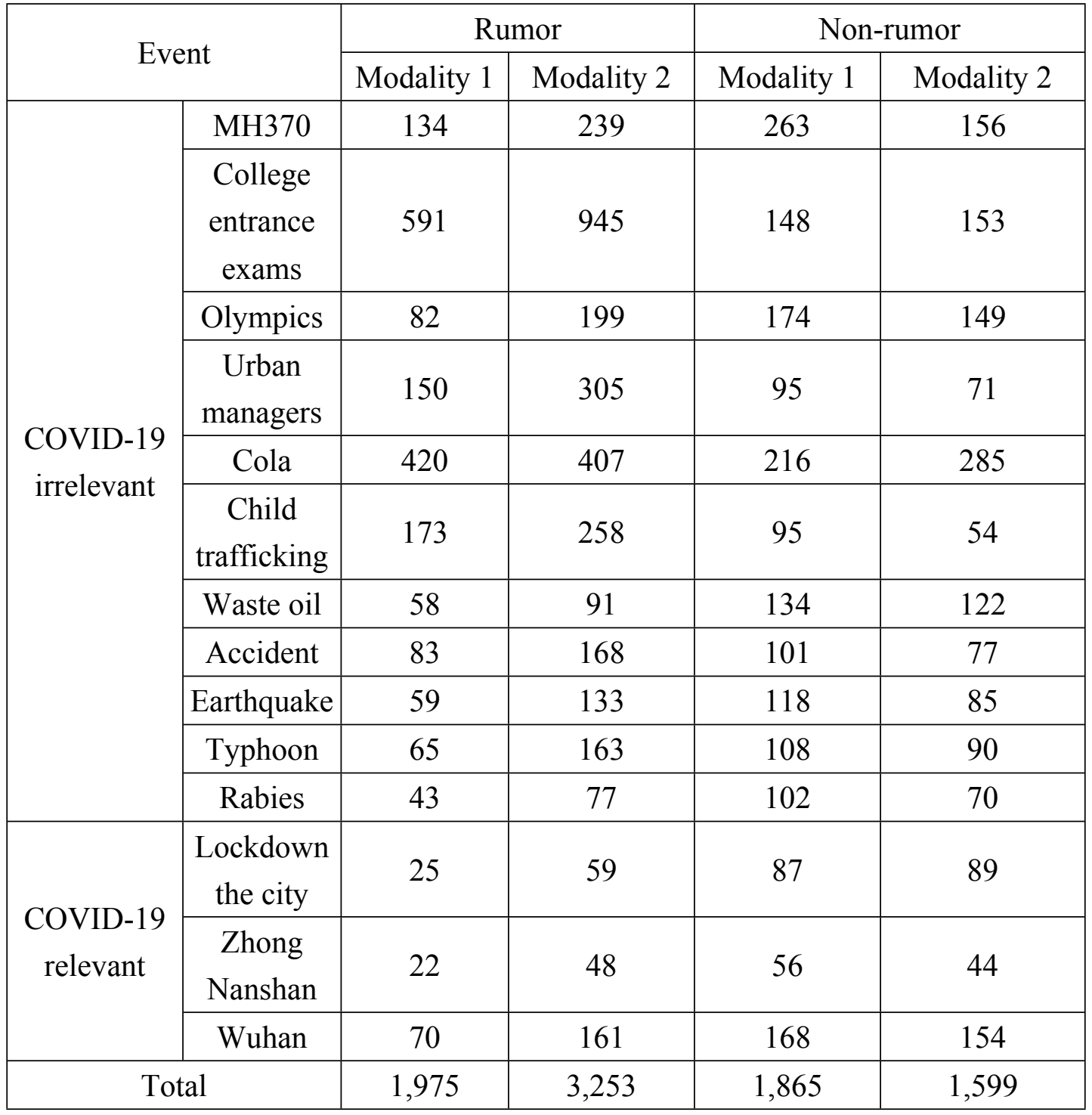




\section{Table 2 (on next page)}

Table 2 Statistics of instances in both datasets for experiments 
1

Table 2 Statistics of instances in both datasets for experiments

\begin{tabular}{|c|c|c|c|c|c|c|}
\hline & \multicolumn{3}{|c|}{ Weibo Dataset } & \multicolumn{3}{|c|}{ PHEME Dataset } \\
\hline & $\begin{array}{c}\text { training } \\
\text { set }\end{array}$ & $\begin{array}{c}\text { validation } \\
\text { set }\end{array}$ & $\begin{array}{l}\text { test } \\
\text { set }\end{array}$ & $\begin{array}{c}\text { training } \\
\text { set }\end{array}$ & $\begin{array}{c}\text { validation } \\
\text { set }\end{array}$ & $\begin{array}{l}\text { test } \\
\text { set }\end{array}$ \\
\hline split 0 & 1,676 & 1,736 & \multirow{3}{*}{428} & 776 & 889 & \multirow{3}{*}{877} \\
\hline split 1 & 2,429 & 983 & & 889 & 776 & \\
\hline split 2 & 2,719 & 693 & & 995 & 889 & \\
\hline $\begin{array}{c}\text { total } \\
\text { number of } \\
\text { instances }\end{array}$ & \multicolumn{3}{|c|}{3,840} & \multicolumn{3}{|c|}{2,207} \\
\hline
\end{tabular}

2 


\section{Table 3 (on next page)}

Table 3 The classification accuracy of Weibo dataset in COVID-19 rumor detection 
1 Table 3 The classification accuracy of Weibo dataset in COVID-19 rumor detection

\begin{tabular}{|c|c|c|c|c|}
\hline & split 0 & split1 & split2 & average \\
\hline DT-EMB & $57.51 \%$ & $56.82 \%$ & $56.47 \%$ & $56.93 \%$ \\
\hline SEQ-CNNs & $66.76 \%$ & $66.89 \%$ & $68.48 \%$ & $67.38 \%$ \\
\hline SEQ-Bi-GRUs & $65.89 \%$ & $71.09 \%$ & $69.81 \%$ & $68.93 \%$ \\
\hline GAN-GRU-early & $60.74 \%$ & $47.09 \%$ & $51.45 \%$ & $53.00 \%$ \\
\hline BiGCN-early & $71.88 \%$ & $63.46 \%$ & 63.50 & $66.28 \%$ \\
\hline COMFUSE-post-only & $73.48 \%$ & $75.22 \%$ & $71.17 \%$ & $73.29 \%$ \\
\hline COMFUSE-com-only & $70.44 \%$ & $74.59 \%$ & $74.11 \%$ & $73.17 \%$ \\
\hline COMFUSE & $\mathbf{7 9 . 1 7 \%}$ & $\mathbf{7 9 . 2 4 \%}$ & $\mathbf{7 7 . 4 1 \%}$ & $\mathbf{7 8 . 6 1 \%}$ \\
\hline
\end{tabular}

2 


\section{Table 4 (on next page)}

Table 4 The classification accuracy of PHEME dataset in latest events rumor detection 
1 Table 4 The classification accuracy of PHEME dataset in latest events rumor detection

\begin{tabular}{|c|c|c|c|c|}
\hline & split 0 & split1 & split2 & average \\
\hline DT-EMB & $56.92 \%$ & $56.67 \%$ & $56.85 \%$ & $56.81 \%$ \\
\hline SEQ-CNNs & $63.06 \%$ & $61.42 \%$ & $63.67 \%$ & $62.72 \%$ \\
\hline SEQ-Bi-GRUs & $63.25 \%$ & $60.83 \%$ & $62.97 \%$ & $62.35 \%$ \\
\hline GAN-GRU-early & $53.47 \%$ & $50.43 \%$ & $56.03 \%$ & $53.31 \%$ \\
\hline BiGCN-early & $67.94 \%$ & $57.94 \%$ & $59.08 \%$ & $61.65 \%$ \\
\hline COMFUSE-post-only & $63.56 \%$ & $65.58 \%$ & $64.17 \%$ & $64.44 \%$ \\
\hline COMFUSE-com-only & $58.36 \%$ & $60.47 \%$ & $57.39 \%$ & $58.74 \%$ \\
\hline COMFUSE & $\mathbf{6 8 . 2 5 \%}$ & $\mathbf{6 6 . 6 7 \%}$ & $\mathbf{6 5 . 3 9 \%}$ & $\mathbf{6 6 . 7 7 \%}$ \\
\hline
\end{tabular}

2 
Figure 1

Figure. 1 Example of the Sina Weibo page, which contains a rumor microblog

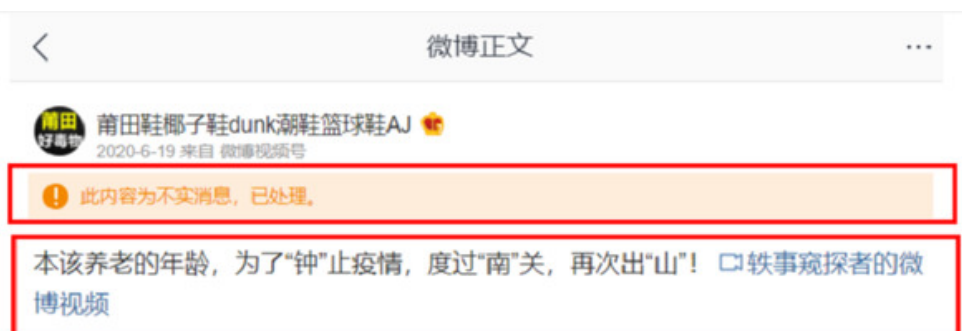
博视频

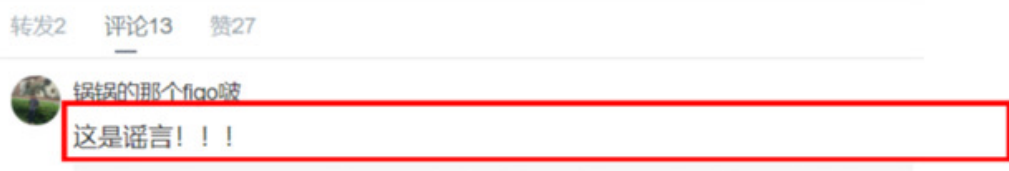

苚田鞋椰子鞋dunk湖鞋篮球鞋AJ:不好意思我也被谣言的了，以后发之前多注意 $2020-6-19$

6. 君陌Ambition

假新闻 昨天就已经辟谣了!

蔽田鞋椰子鞋dunk湖鞋篮球鞋AJ:这样明我的错

2020-6-19

P皮皮小垪于18

澄清了没有去北京 在广州

荒田鞋椰子鞋dunk潮鞋篮球鞋AJ:谢谢提醒

2020-6-19

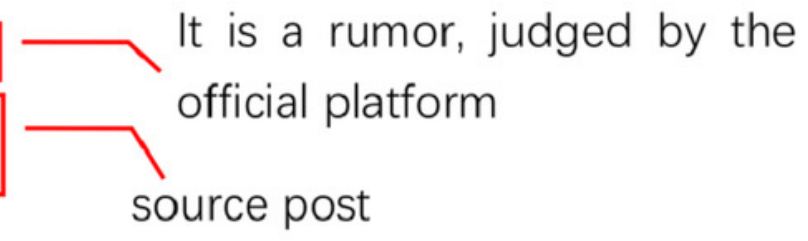

comment 1

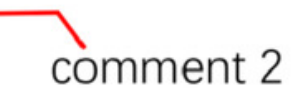

B1

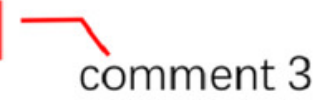


Figure 2

Figure. 2 Workflow of the rumor judgement by the official Sina Weibo community management center

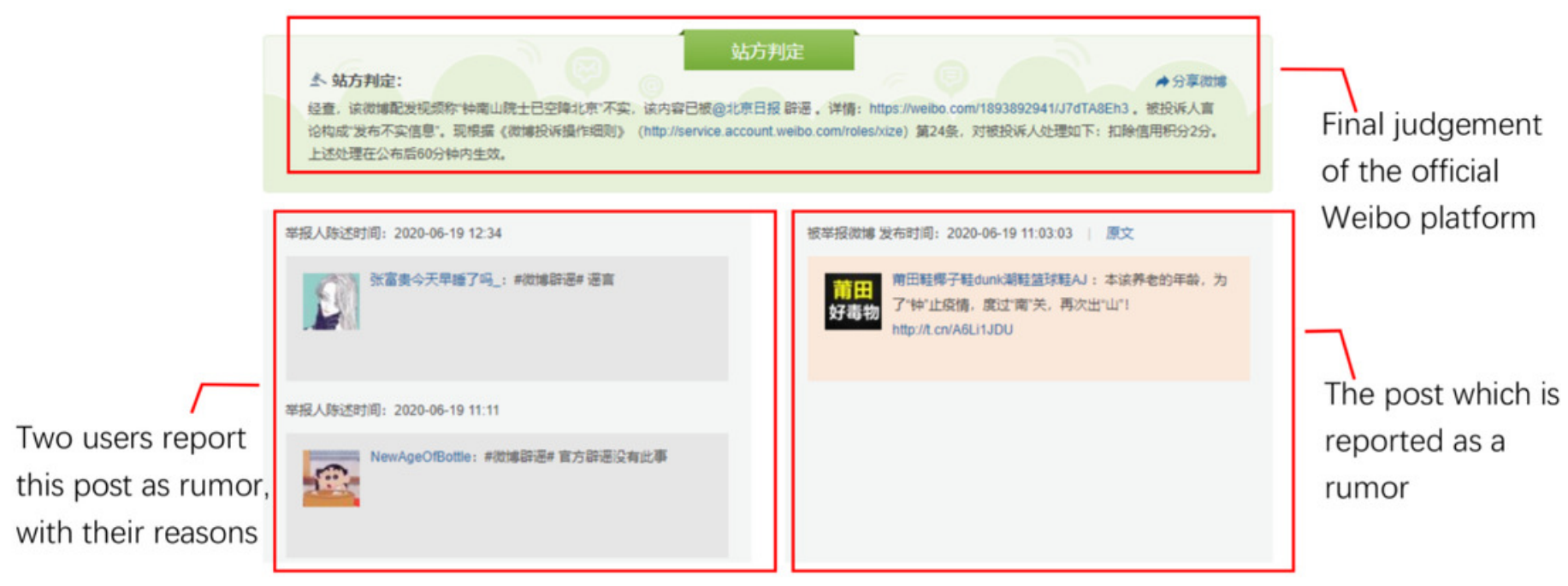


Figure 3

Figure. 3 Workflow of COMFUSE

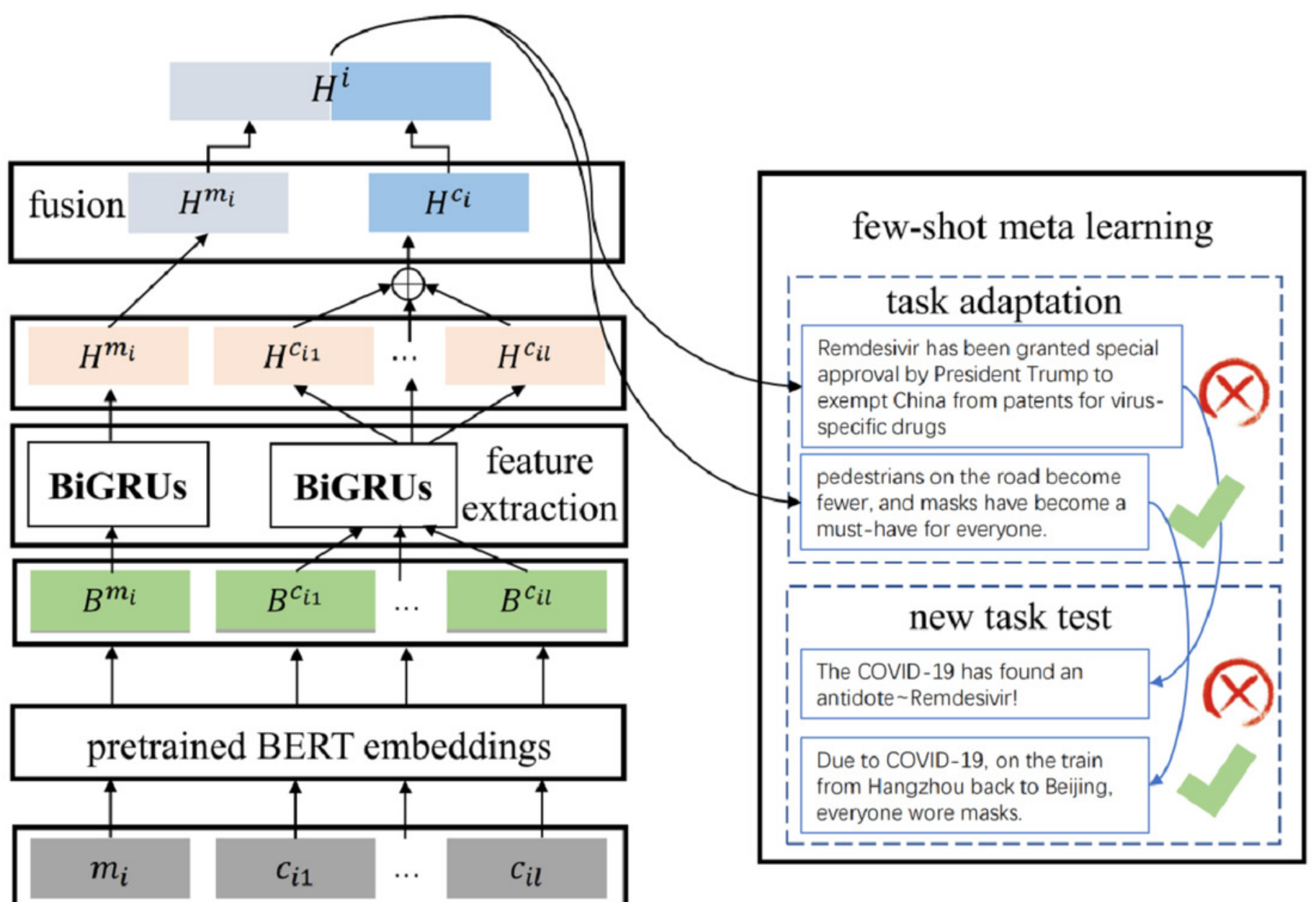


Figure 4

Figure. 4 Illustrations of word embeddings with BERT

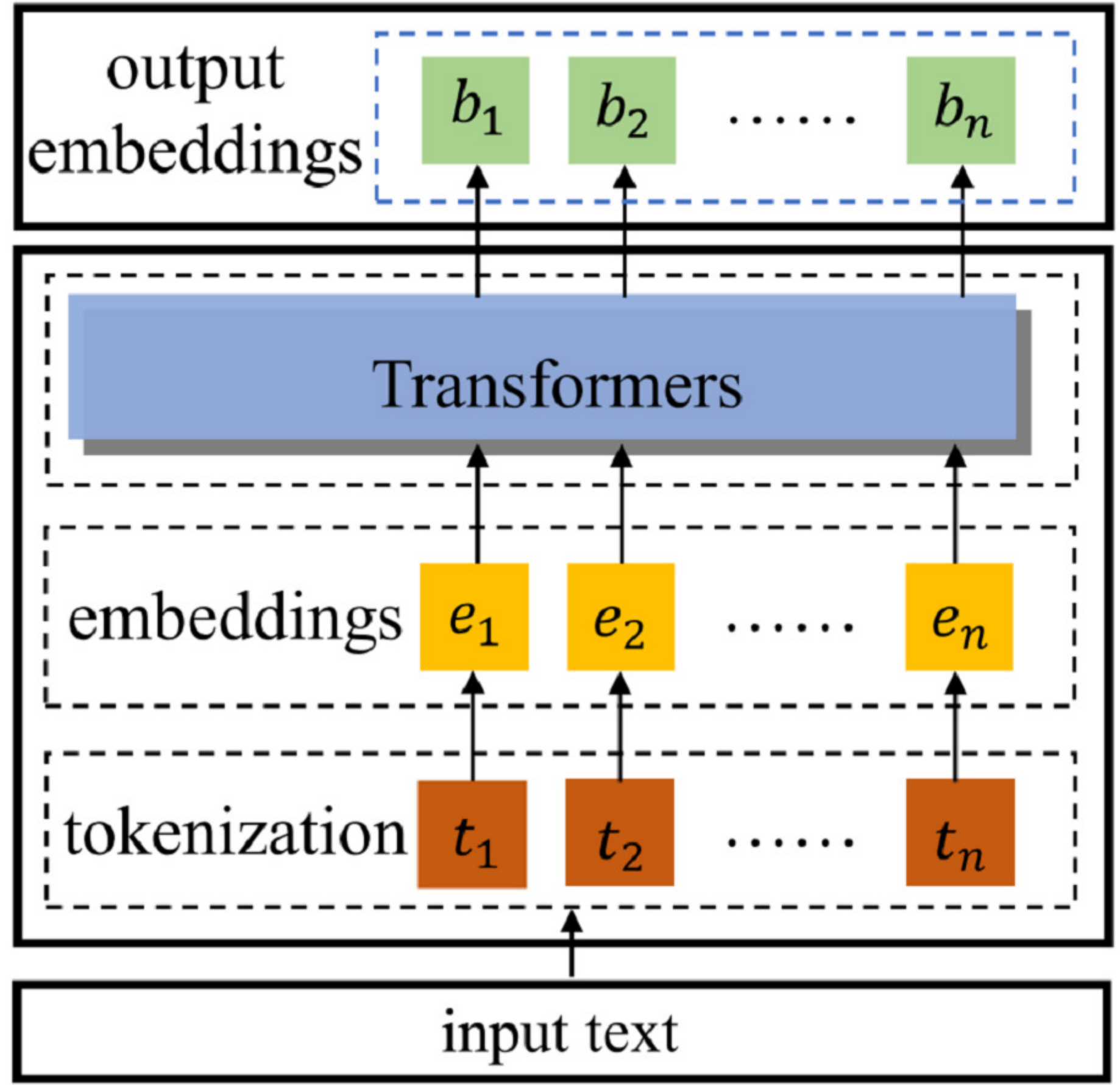


Figure 5

Figure. 5 Structure of Bi-GRUs 


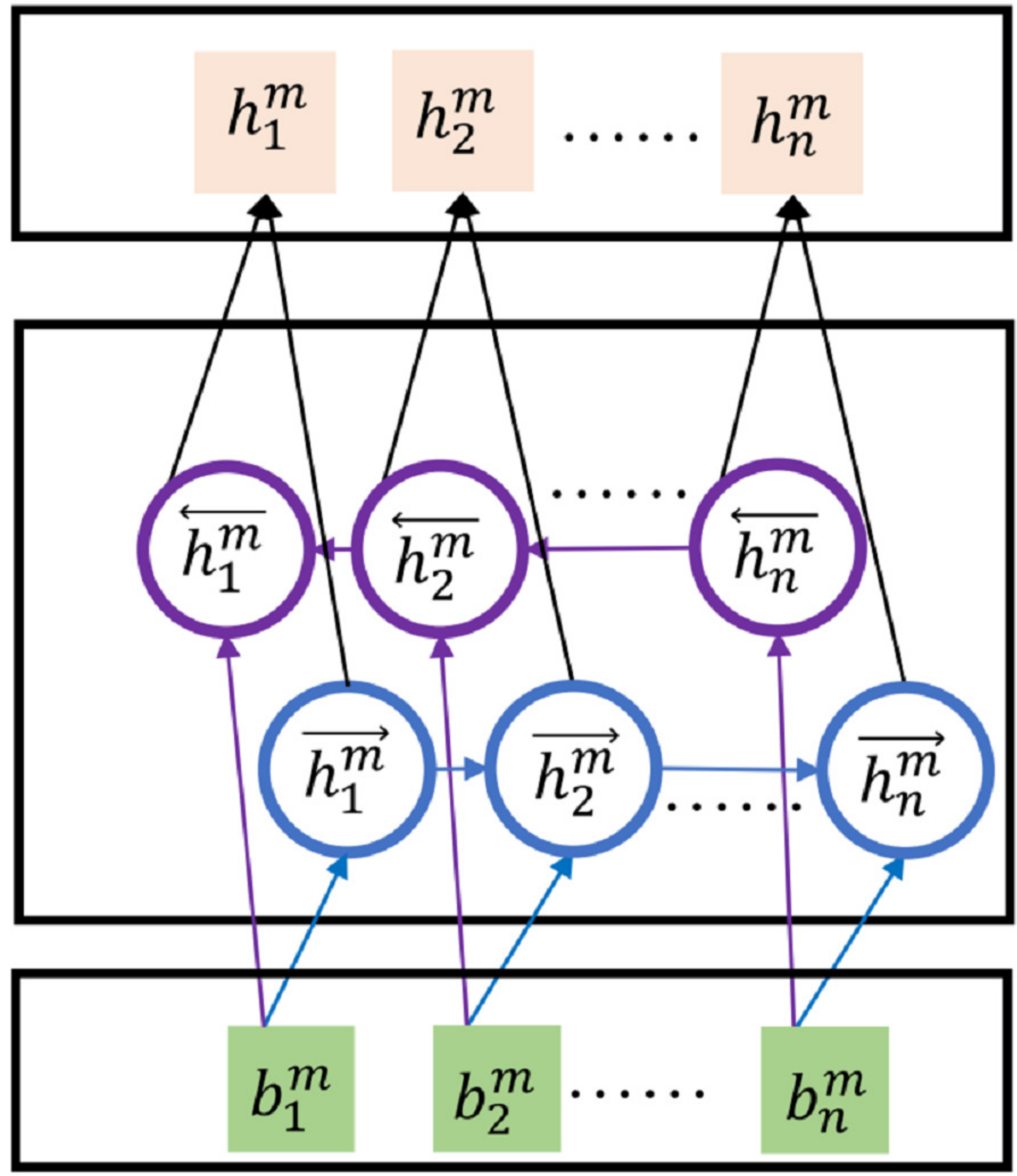


Figure 6

Figure. 6 Workflow of one meta-learning iteration

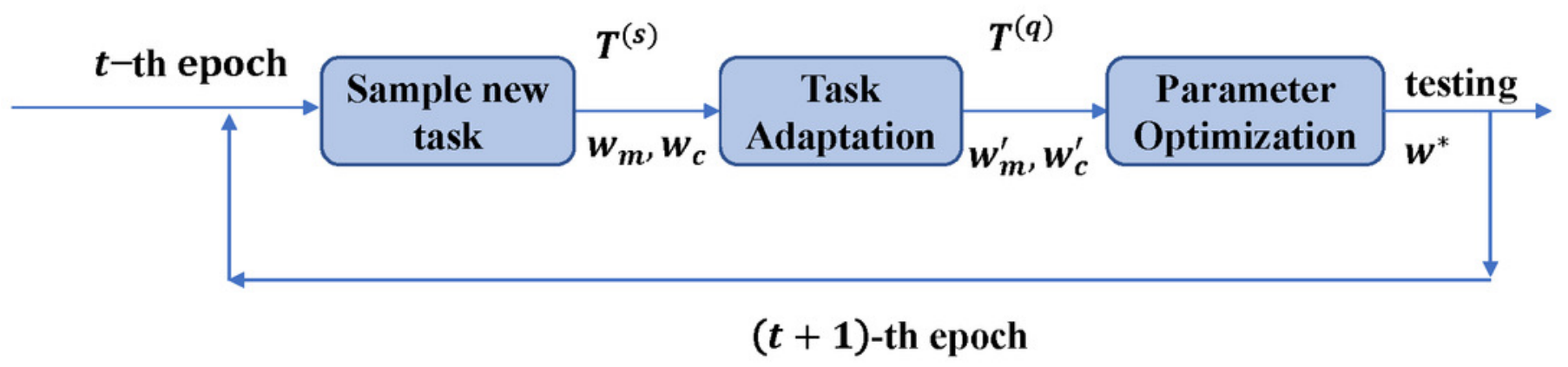


Figure 7

Figure. 7 Statistics of length per text of the Weibo dataset

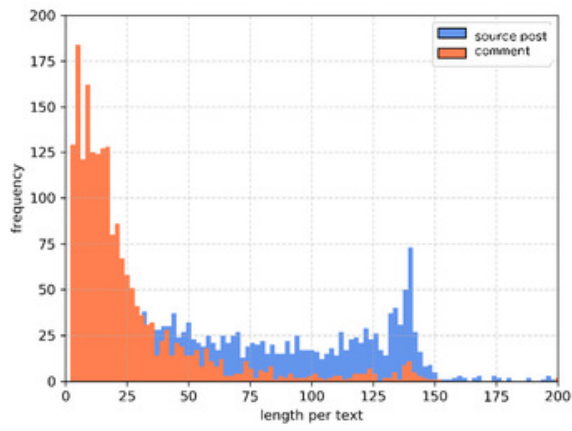

a. training set of split 0

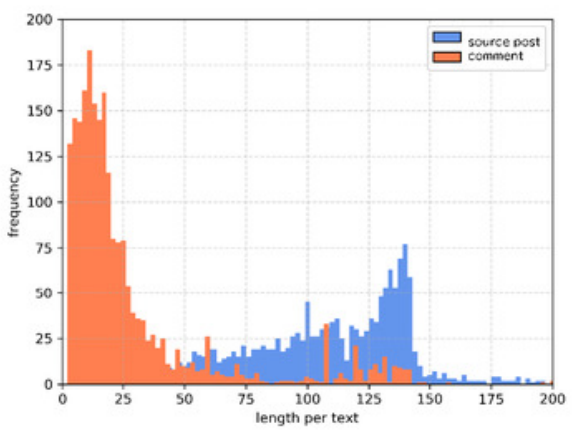

d. validation set of split 0

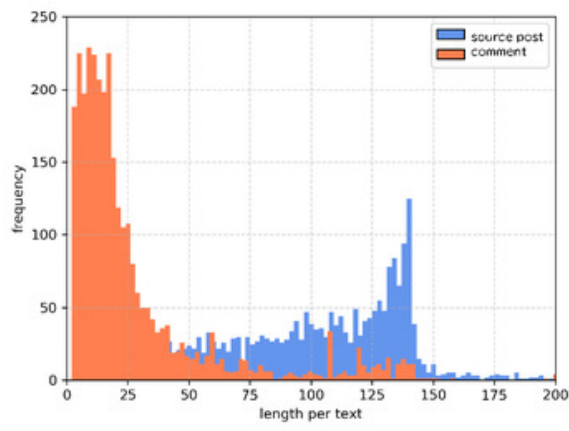

b. training set of split 1

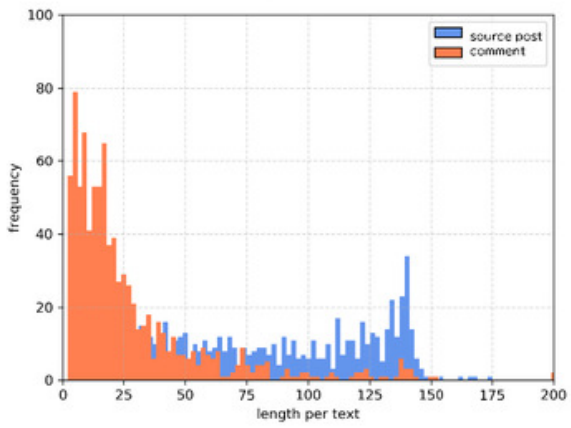

e. validation set of split 1

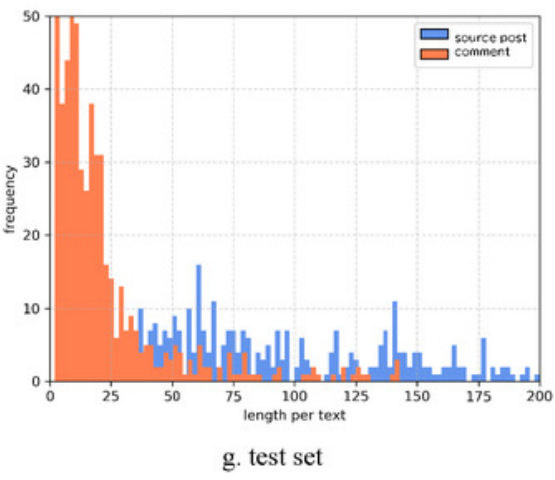

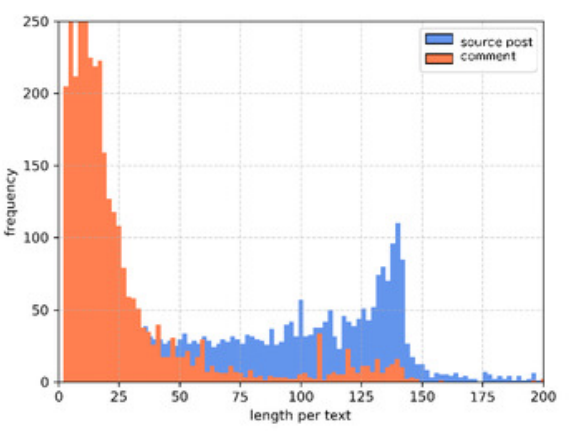

c. training set of split 2

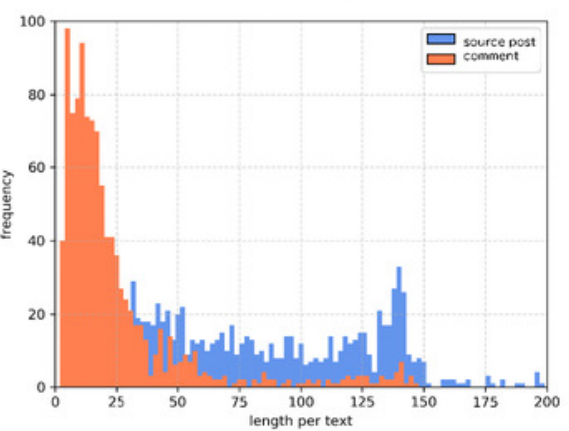

f. validation set of split 2 
Figure 8

Figure. 8 Statistics of length per text of the PHEME dataset

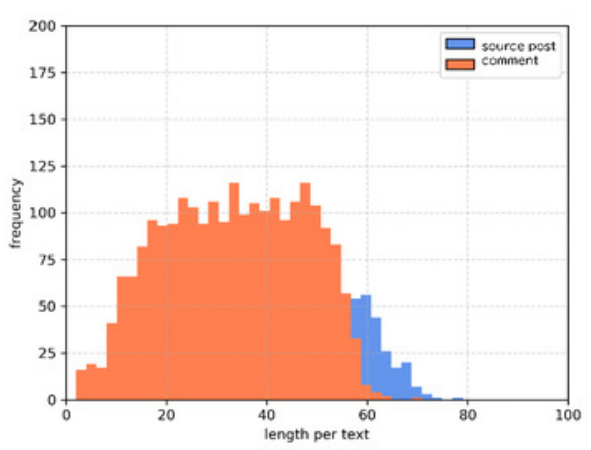

a. training set of split 0

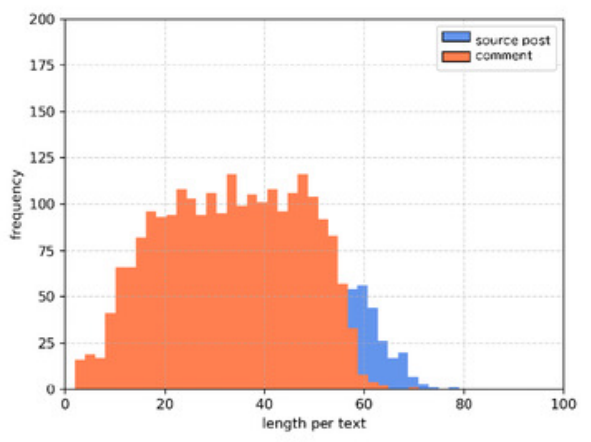

d. validation set of split 0

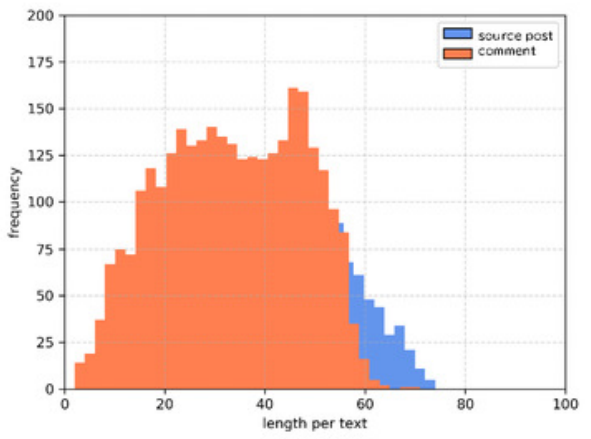

b. training set of split 1

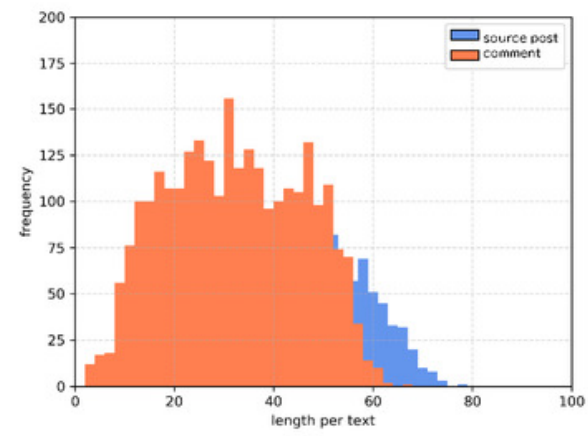

e. validation set of split 1

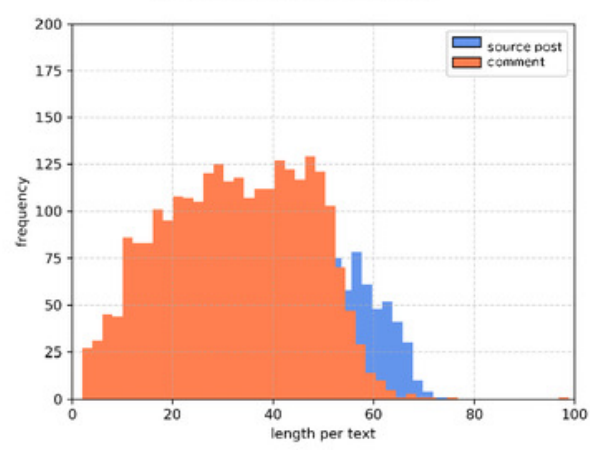

g. test set

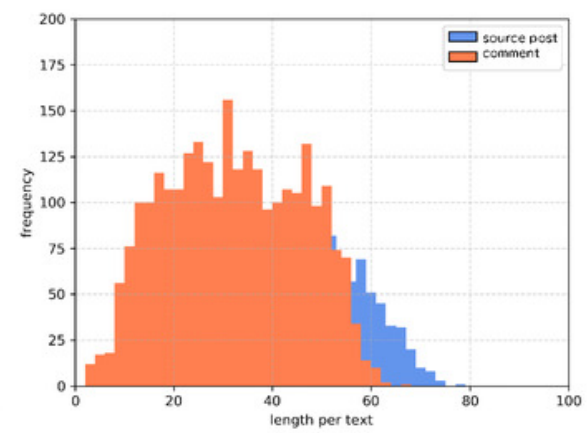

c. training set of split 2

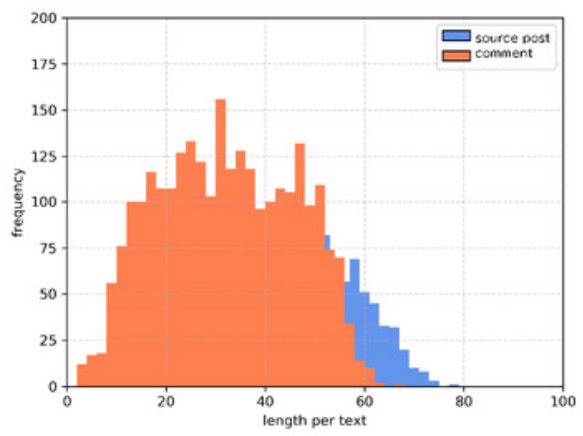

f. validation set of split 2 
Figure 9

Figure. 9 Experimental results of different pad sizes of source posts with a fixed pad size of comments as 32 on the Weibo dataset

$$
\multimap \text { split } 0 \multimap \text { split } 1 \multimap \text { split } 2 \multimap \text { average }
$$

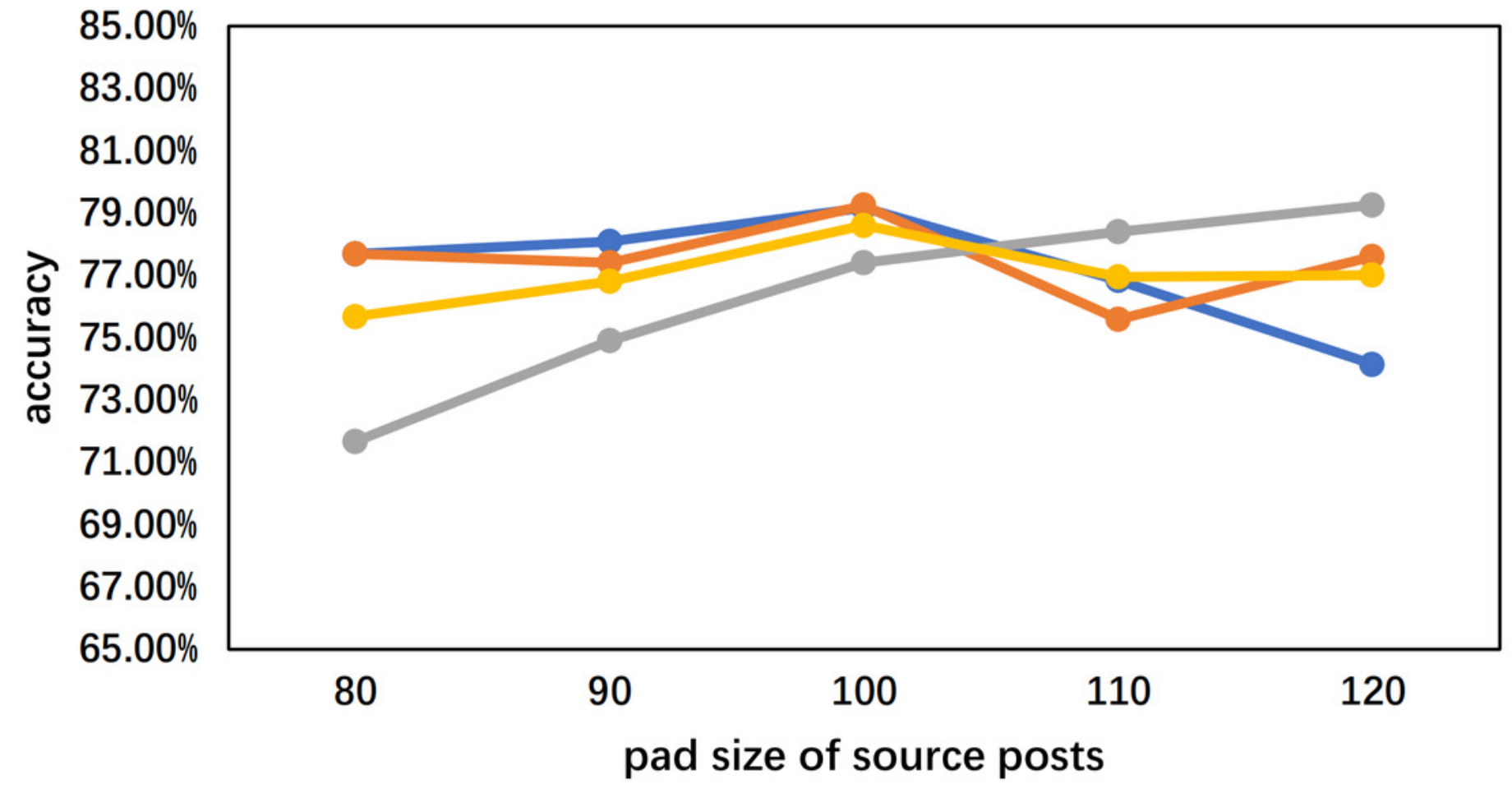


Figure 10

Figure. 10 Experimental results of different pad sizes of comments with a fixed pad size of source posts as 100 on the Weibo dataset

\section{$\multimap$ split $0 \multimap$ split $1-$ split $2 \multimap$ average}

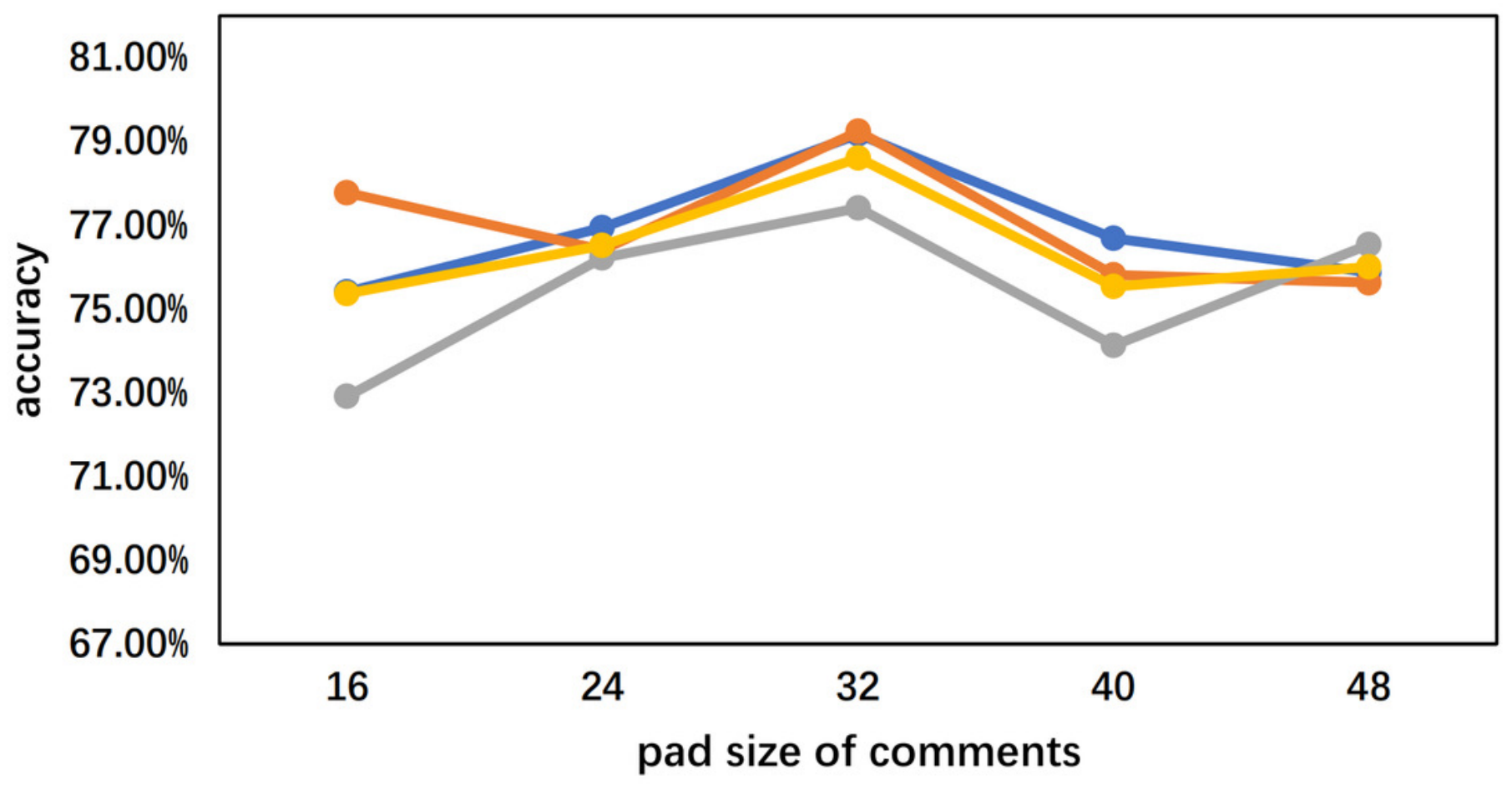

